\title{
Measuring Interatomic Bonding and Charge Redistributions in Defects by Combining 4D-STEM and STEM Multislice Simulations
}

\author{
Damien Heimes, Jürgen Belz, Andreas Beyer and Kerstin Volz
}

Faculty of Physics and Materials Sciences Center, Philipps-Universität Marburg, Marburg, Hessen, Germany

The dynamical properties of charge carriers in electronic devices, i.e. electrons and holes, are strongly influenced by electric fields formed inside the devices. Crystal imperfections like charged wrong bonds therefore can affect the efficiency of a device to a great extent. This makes it necessary to understand and characterize materials on an atomic level accounting for interatomic bonding. Since electrons are deflected by electric fields inside the specimen, scanning transmission electron microscopy (STEM) with probe sizes in the sub-Angstrom regime is a suitable technique for this.

The momentum transfer induced by electric fields is directly proportional to the shift in the center of mass $(\mathrm{COM})$ of the diffraction pattern in STEM measurements $[1,2]$. Fast pixelated detectors make it possible to record the whole diffraction pattern while scanning the beam. Hence the COM shift becomes experimentally accessible and it opens up the possibility to determine electric fields and charge densities on an atomic level $[3,4]$.

Besides electric fields in the specimen there also are many more factors influencing the COM shift in the diffraction pattern. The influence of all those effects overlap in experiment, so a COM shift does not necessarily prove the existence of an electric field.

In order to quantify electric fields from atomic bonding using STEM, we apply STEM multislice simulations $[6,7]$, in which we are able to investigate the influence of single quantities by varying them over a certain range while keeping all other parameters fixed. This is the great advantage of simulations over the experiment.

As a first case study we will use STEM simulations to investigate the charge redistribution at a crystal defect called anti-phase boundary. This kind of defect appears for example in gallium phosphide (GaP), when it is grown on a silicon ( $\mathrm{Si}$ ) substrate [7]. Monoatomic steps in the $\mathrm{Si}$ cause a change in polarity in the GaP, illustrated in Figure 1. An anti phase is formed, in which the occupation of the atomic sites is interchanged compared to the main phase. At the boundary from one phase to the other (the anti phase boundary (APB)), charged wrong bonds of Ga-Ga and P-P are formed [7] (highlighted in red in Figure 1).

To illustrate the results the simulations can generate, Figure 2 shows the simulated COM in $\mathrm{x}$-direction for $\mathrm{GaP}$. The position of atoms is shown by crosses. One can clearly see that the center of mass is shifted towards the atomic positions.

The fraction on the COM shift which stems from the charged wrong bonds will be determined by comparing simulations using isolated atoms scattering potentials, neglecting atomic bonding, with simulations which include the charge redistribution due to bonding by calculating the scattering potentials via density functional theory (DFT). Similar to [8] the potentials are calculated using the GPAW DFT code [9]. 
Other effects which affect the COM shift will also be investigated, beginning with the specimen mistilt, because this is likely to happen in experiment.

We aim to show that our method, i.e. making use both of STEM simulations including DFT scattering potentials and 4D-STEM data, enables us to measure interatomic bonding and by that determine the charge of wrong bond defects.

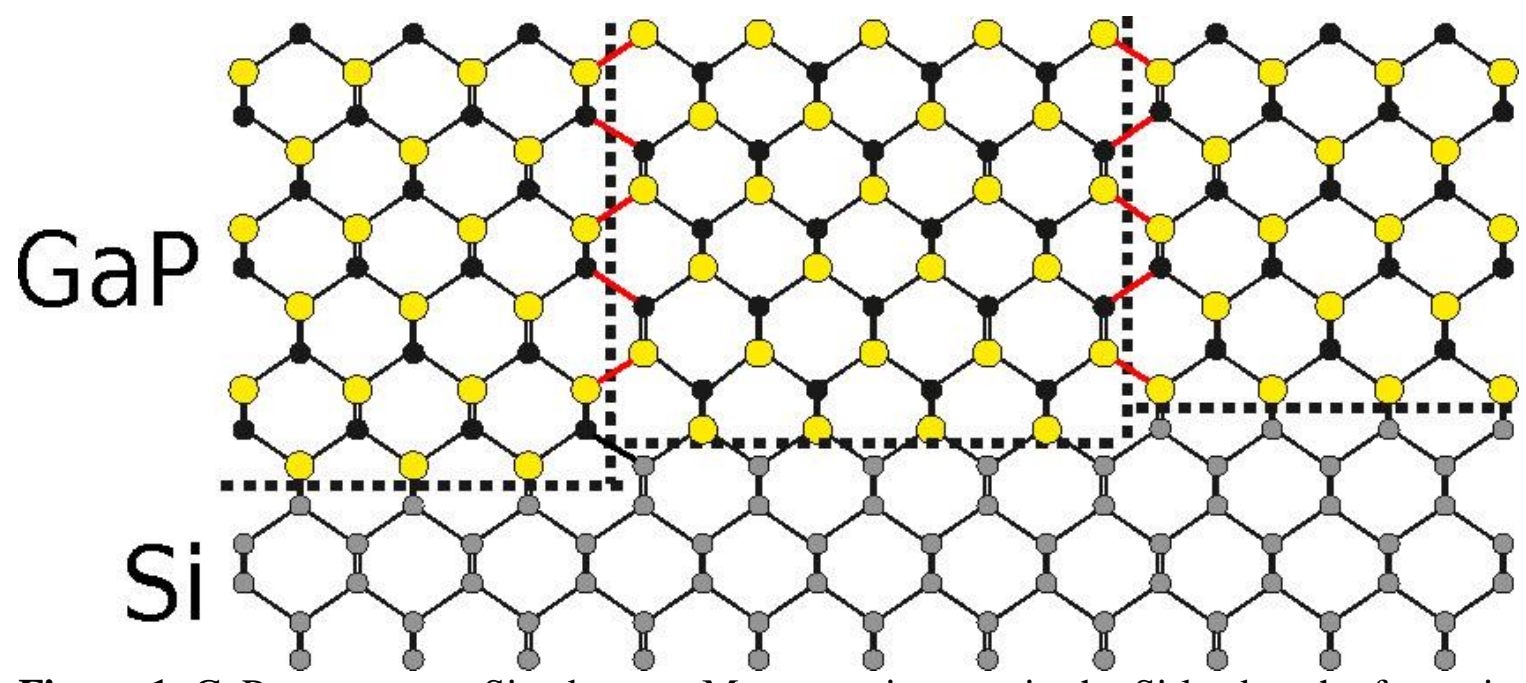

Figure 1. GaP grown on a Si substrate. Monoatomic steps in the Si lead to the formation of anti-phase boundaries (APB) in the GaP, illustrated with vertical dashed lines. In the main phase and anti phase, the positions of $\mathrm{Ga}$ and $\mathrm{P}$ are interchanged. The resulting charged wrong bonds, which form at the APBs, are marked in red.
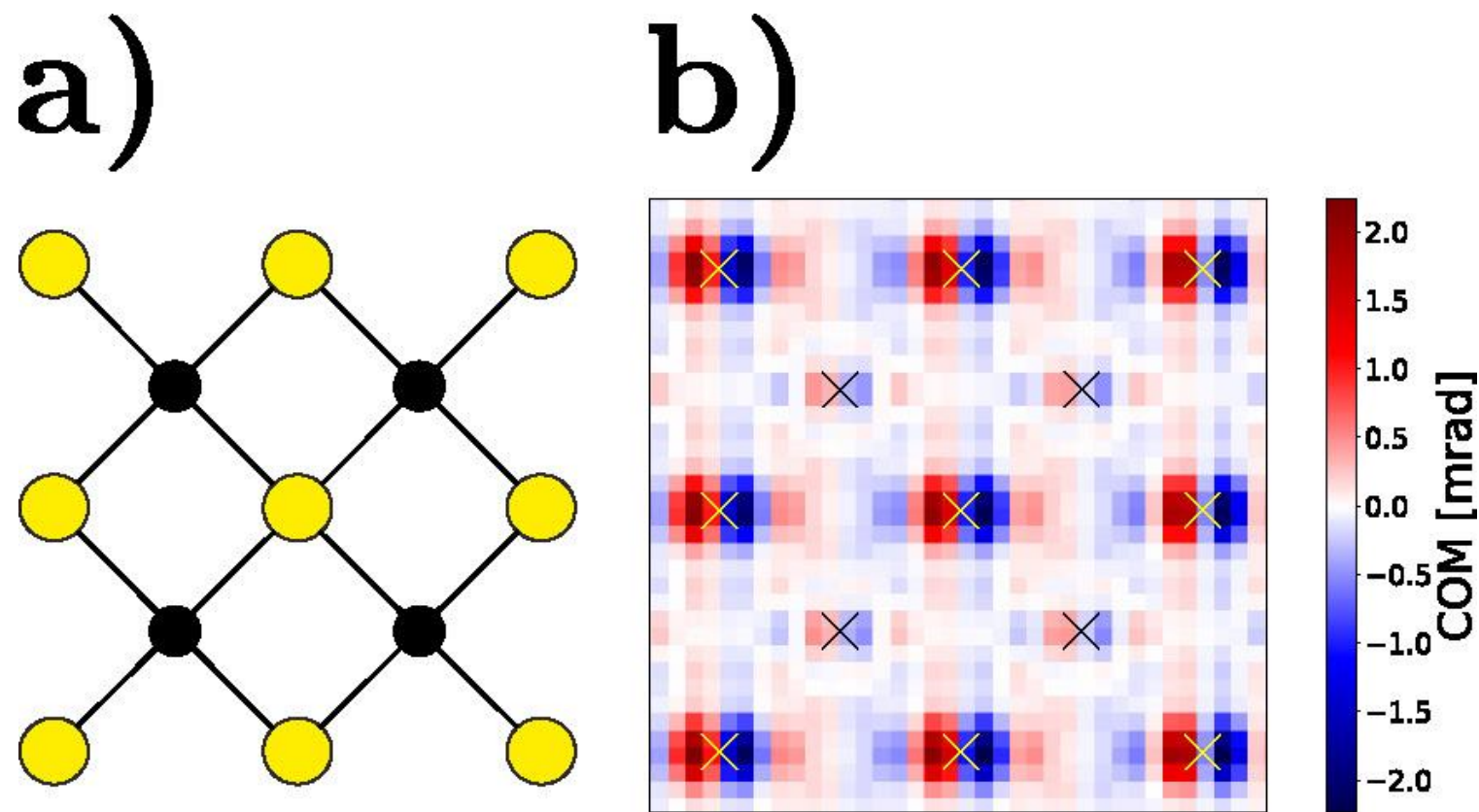

Figure 2. a) Cubic unit cell of $\mathrm{GaP}$ in [001] viewing direction. $\mathrm{Ga}$ atoms are shown in yellow, $\mathrm{P}$ atoms in black. b) Center of mass (COM) in x-direction for one cubic unit cell of GaP from STEM multislice simulations. Crosses show the positions of atoms with the same color code as in a). 


\section{References}

[1] Müller, Knut, et al. "Atomic electric fields revealed by a quantum mechanical approach to electron picodiffraction." Nature communications 5.1 (2014): 1-8.

[2] Müller-Caspary, Knut, et al. "Measurement of atomic electric fields and charge densities from average momentum transfers using scanning transmission electron microscopy." Ultramicroscopy 178 (2017): 62-80. [3] Shibata, Naoya, et al. "Electric field imaging of single atoms." Nature communications 8.1 (2017): 1-7.

[4] Gao, Wenpei, et al. "Real-space charge-density imaging with sub-ångström resolution by four-dimensional electron microscopy." Nature 575.7783 (2019): 480-484.

[5] Kirkland, Earl J. Advanced computing in electron microscopy. Springer Science \& Business Media, 2010. [6] Oelerich, Jan Oliver, et al. "STEMsalabim: a high-performance computing cluster friendly code for scanning transmission electron microscopy image simulations of thin specimens." Ultramicroscopy 177 (2017): 91-96.

[7] Beyer, A., et al. "Atomic structure of (110) anti-phase boundaries in GaP on Si (001)." Applied Physics Letters 103.3 (2013): 032107.

[8] Susi, Toma, et al. "Efficient first principles simulation of electron scattering factors for transmission electron microscopy."Ultramicroscopy 197 (2019): 16-22.

[9] Enkovaara, J. E., et al. "Electronic structure calculations with GPAW: a real-space implementation of the projector augmented-wave method." Journal of Physics: Condensed Matter 22.25 (2010): 253202. 